\title{
Commentary
}

\section{Extrapolation of the Animal Carcinogenesis Threshold to Humans ${ }^{1}$}

\author{
Minako Nagao ${ }^{2,3,5}$, Satoko Ishikawa ${ }^{2}$, Hitoshi Nakagama ${ }^{3}$ and Masahiko Watanabe ${ }^{4}$ \\ ${ }^{2}$ Keio University Faculty of Pharmacy, Tokyo Japan \\ ${ }^{3}$ National Cancer Center Research Institute, Tokyo Japan \\ ${ }^{4}$ School of Pharmacy, Shujitsu University, Okayama, Japan
}

(Received September 18, 2008; Revised October 14, 2008; Accepted October 16, 2008)

The presence or absence of a threshold in carcinogenesis for genotoxic carcinogens was reevaluated. The ED01 study of 2-acetylaminofluorene, performed in the U.S. using more than 24,000 mice, provides us with information about the practical limits of an attainable experimental approach for determining carcinogenesis thresholds. The data indicated that the dose response was highly nonlinear and an apparent threshold existed for bladder carcinogenesis, but that it was linear-no-threshold for liver carcinogenesis in the same animals. Despite smaller study sizes, we attempted to evaluate the carcinogenesis dose response to 2-amino-1-methyl-6-phenylimidazo[4,5-b]pyridine (PhIP) and 2-amino-3,8-dimethylimidazo[4,5-f]quinoxaline (MelQx) in rats, using published data. Mammary tumors were induced in female F344 rats by PhIP in a linear-no-threshold dose response, as was lymphocytic leukemia in male and female rats. However, colon tumors were induced in a non-linear dose response, possibly with a threshold, in the same male animals. Liver tumors were induced in male F344 rats by MelOx, and preneoplastic changes in the liver were induced in non-linear dose response, possibly with a threshold. From these findings, it can be deduced that linear or non-linear dose response with or without thresholds changes depending upon the exposing chemical, species and target organs. Considering heterogeneity of humans there would be no appropriate animal models to evaluate threshold in humans for carcinogenicity of chemicals.

Some types of genotoxic carcinogens, such as methyl methanesulfonate, ethyl methanesulfonate and $\mathbf{N}$-methyl$N^{\prime}$-nitro- $N$-nitrosoguanidine, show highly non-linear dose response with an apparent threshold in mammalian cells or bacteria in vitro. Involvement of repair mechanisms strongly supports the presence of a threshold. Although it is necessary to confirm non-linear dose response for animal carcinogenesis of a compound showing a threshold for in vivo genotoxicity, it is expected that such compounds exhibit thresholds for human carcinogenesis. Dose response studies of genotoxic carcinogens will provide information on using valuable chemicals safely.

Key words: genotoxic carcinogen, threshold, dose response, linear-no-threshold, non-linear, target organ

\section{Introduction}

There is a movement to review the current risk evaluations of genotoxic carcinogens that there is no threshold for hazard effect. Since biological systems have DNA repair mechanisms, it can be speculated that there should be thresholds for mutagenesis and carcinogenesis induced by genotoxic carcinogens.

Extensive studies were performed approximately 30 years ago in the U.S. to clarify the threshold for carcinogenesis by a genotoxic carcinogen, 2-acetylaminofluorene (AAF) evaluating more than 24,000 mice $(1,2)$. Highly non-linear dose response to AAF with an apparent threshold was demonstrated for bladder tumors in female BALB/c mice, but linear dose response without threshold for liver tumors in the same animals. On the other hand, linear dose responses were found in DNAAAF adduct formations in the bladder and liver of $\mathrm{BALB} / \mathrm{c}$ mice (3). Linear-no-threshold dose response for mutagenesis is generally accepted.

Recently, it was claimed that there are thresholds for carcinogenesis, preneoplastic changes, and mutagenicity when using heterocyclic amines (HCAs) (4-6). These investigators attempted to demonstrate the presence of thresholds using very low doses, including that comparable to human HCA exposure.

In the present article, we introduce the AAF-ED01 study. In this study, we compared the mode of carcinogenesis dose response of two mutagen-carcinogen HCAs, 2-amino-1-methy-6-phenylimidazo[4,5-b]pyridine (PhIP) and 2-amino-3,8-dimethylimidazo[4,5$f$ ]quinoxaline (MeIQx). Existing HCA experimental data were not sufficient to evaluate presence or absence of thresholds, so we compared the mode of dose response in inducing neoplastic change in different organs of the same animals. Even using small numbers of

${ }^{1}$ Presented at the International Symposium on Genotoxic and Carcinogenic Thresholds, Tokyo, July 22/23, 2008

${ }^{5}$ Correspondence to: Minako Nagao, Keio University Faculty of Pharmacy, 1-5-30 Shibakoen Minato-ku, Tokyo 105-8512, Japan. Tel/Fax: +81-45-822-1081, E-mail: mnagao@m8.dion.ne.jp 
animals, we found that PhIP induced mammary tumors and leukemia in a linear-no-threshold dose response, and it induced colon tumors in a non-linear dose response with a possible threshold (7). As seen in the AAF carcinogenesis study, the PhIP carcinogenesis study indicated that the mode of dose response differed depending upon the target organs. In this article, we present published scientific data that is necessary to understand how to regulate genotoxic carcinogens correctly.

Genotoxic carcinogens are generally considered to have no threshold for genotoxicity. In this study, we really confirmed the absence of thresholds for in vivo genotoxicity for several compounds including HCAs by use of published data $(8,9)$. However, some DNA-reacting genotoxic agents were demonstrated to show highly non-linear dose responses in vitro, with an apparent threshold $(10,11)$. Involvement of repair mechanisms was indicated for this non-linearity (11), and it was calculated that the repair was about $99.99 \%$ below the threshold (19). Therefore, it is reasonable to expect that there are thresholds for carcinogenesis of these compounds. By clarifying genotoxicity dose response of chemicals concerned, some genotoxic chemicals can be utilized below the threshold, safely.

\section{Information Obtained from Dose-response Study in AAF Tumorigenesis}

We plotted the reported data of the ED01 study of AAF (CAS 53-96-3) (24,192 female Balb/c mice with lifetime dosing) (12), on an arithmetic scale (Fig. 1). It indicates the presence of an apparent threshold for bladder tumors with a highly non-linear dose response and an absence of threshold for liver tumors when the incidence was extrapolated from doses where a significant increase was observed. After 33 months of treatment, the dose response curve for bladder tumors showed a semi-logarithmic fit with an apparent threshold (Fig. 1a), as reported by Waddell (13). The equation and correlation coefficient we obtained are $\mathrm{y}=112.37 \mathrm{Ln}(\mathrm{x})-$ 460.77, $\mathrm{R}=0.98$. However, at 18 and 24 months, linear fit was seen with apparent thresholds. The logarithmic fit for bladder carcinogenesis may be due to saturation in incidence with $150 \mathrm{ppm}$ dosing at 33 months, and linear dose response with an apparent threshold was estimated below $100 \mathrm{ppm}$. The apparent threshold (the point where the line crossed zero) decreased with increased treatment duration, as pointed out previously (13).

For liver carcinogenesis, the dose response curve over 33 months showed a linear fit (Fig. 1b). The apparent threshold was calculated to be $3 \mathrm{ppm}(\mathrm{y}=0.47 \mathrm{x}-1.42$, $R=0.97)$. The apparent threshold values decreased with increased treatment duration, and we calculated it to be $0 \mathrm{ppm}$ by 34 months, by use of an equation deduced by Waddell (13). Thus, there would be no threshold, as was also previously reported $(12,14)$. In organs where tumors develop spontaneously, carcinogens may show linear dose responses without thresholds. Background tumor incidences in the liver were $1.1 \%, 2.6 \%$, and 17 $\%$, at 18,24 , and 33 months, respectively, compared with $0.4 \%, 0.3 \%$, and $1 \%$ in the bladder at 18,24 , and 33 months (12).

In this article, the dose response relationship for bladder carcinogenesis is designated as nonlinear and that for liver carcinogenesis as linear-no-threshold.

\section{Information Obtained from Dose Response Studies of HCA Induced Neoplastic Changes}

Dose response studies of PhIP (CAS 105650-23-5) and MeIQx (CAS 77500-04-0) carcinogenesis have been performed with limited numbers of animals and selected dose groups. However, since these compounds induce malignant changes in multiple organs, analysis of experimental data would be useful to help define whether the dose response curves are dependent on target or-
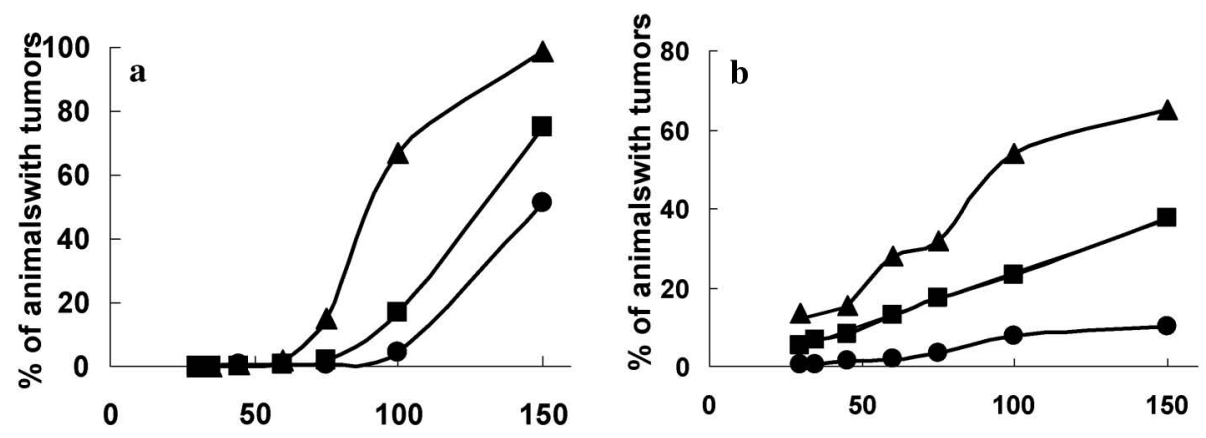

\section{AAF in diet (ppm)}

Fig. 1. Summary of ED01 study. Percent of Balb/c mice with tumors, after administration of diet containing AAF for $18(\bullet)$, $24(\bullet)$, or 33 months $(\mathbf{\Lambda})$. The values of animals with tumors (\%) were subtracted values with that of control. Experimental data were from (12). a: Bladder tumor; $\mathbf{b}$ : Liver tumor. 

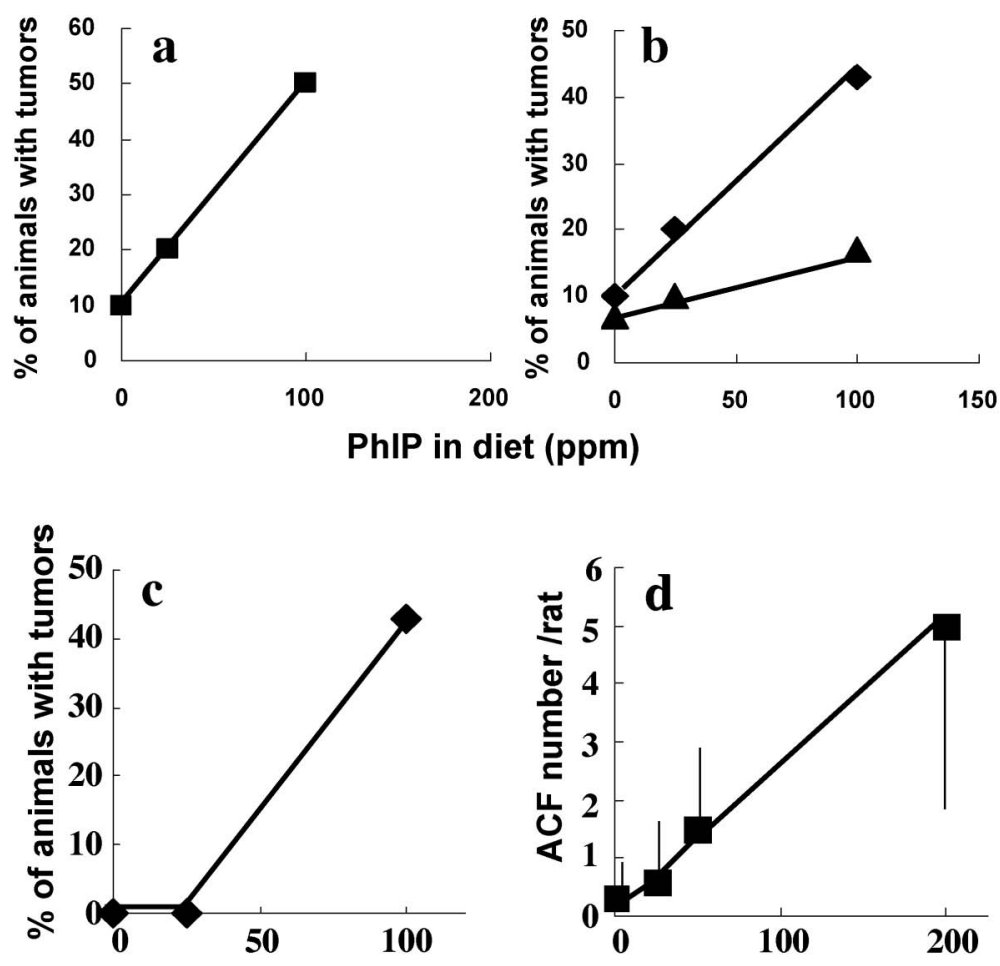

PhIP in diet (ppm)

Fig. 2. Dose effect of PhIP on neoplastic or preneoplastic changes in rats. a: Mammary tumors after 104-week administration to female F344 rats. Data were from (7). b: Lymphatic leukemia after 104-week administration to F344 rats. Data were from (7). 口 : Male, $\mathbf{1}$ : Female. c: Colon tumors after 104-week administration to male F344 rats. Data were from (7). d: ACF after 16-week administration to male F344 rats. Vertical bar indicates standard deviation. Data were from (4).

gans. Published data show that PhIP induces tumors in mammary glands, the hematopoietic system, colon, and prostate in rats $(7,15)$. MeIQx induces tumors in the liver, skin, Zymbal glands, and clitoral glands of rats $(16,17)$. To estimate the shape of the dose response, all experimental data including those which did not show significant difference from the control group were included, except where indicated.

PhIP-mammary tumorigenesis: Hasegawa et al. (7) reported a linear dose response to PhIP dosing using female F344 rats. Each group of 30 rats was fed a diet containing 0,25 , or $100 \mathrm{ppm}$ of PhIP for 104 weeks. Mammary tumor incidences (adenoma or carcinoma) were $10 \%, 20 \%$, and $50 \%$ in the 0,25 , and $100 \mathrm{ppm}$ groups, respectively; the dose response was linear-nothreshold (Fig. 2a), fitting to $y=4 x+10$, with correlation coefficient, $R=1$. In their experiment, a statistically significant increase was observed only for the $100 \mathrm{ppm}$ group.

PhIP-leukemogenesis: Lymphocytic leukemia was induced in male and female F344 rats by feeding PhIP for 104 weeks (7). The incidences of leukemia were 10 $\%, 20 \%$, and $43 \%$ in male rats, and $7 \%, 10 \%$, and $17 \%$ in female rats, with diets containing 0,25 , and $100 \mathrm{ppm}$ of PhIP, respectively (Fig. 2b). The dose response was liner-no-threshold, fitting to $\mathrm{y}=0.33 \mathrm{x}+10.8$, with $\mathrm{R}=$ 0.998 for the male rats, and $y=0.097 x+7.1$, with $R=$ 0.996 for female rats. The control group incidences were $10 \%$ and $7 \%$ in male and female rats, respectively. A statistically significant increase was observed only with $100 \mathrm{ppm}$ group of male rats.

PhIP-colon tumorigenesis: Colon tumors (all carcinomas, no adenomas were observed) were induced in male F344 rats, by feeding PhIP in diet at 0, 25, and 100 ppm for 104 weeks (7). The dose response was nonlinear, with tumor incidences of $43 \%$ at $100 \mathrm{ppm}$ and $0 \%$ at $0 \mathrm{ppm}$ or $25 \mathrm{ppm}$ (Fig. 2c). A possible threshold was indicated.

PhIP-colon preneoplastic change: The dose effect of PhIP in preneoplastic changes was examined by Fukushima et al. (4). They analyzed aberrant crypt focus (ACF) formation after feeding PhIP in the diet at $0,0.001,0.01,0.1,110,50,100$, or $400 \mathrm{ppm}$ for 16 weeks, and claimed that there was a threshold (4). They used 61-244 rats for each group, but the standard deviation for the ACF number/rat was very large [2 or more coefficients of variation (standard deviation/average) in the groups receiving 0 to $10 \mathrm{ppm}$ ] where no significant 


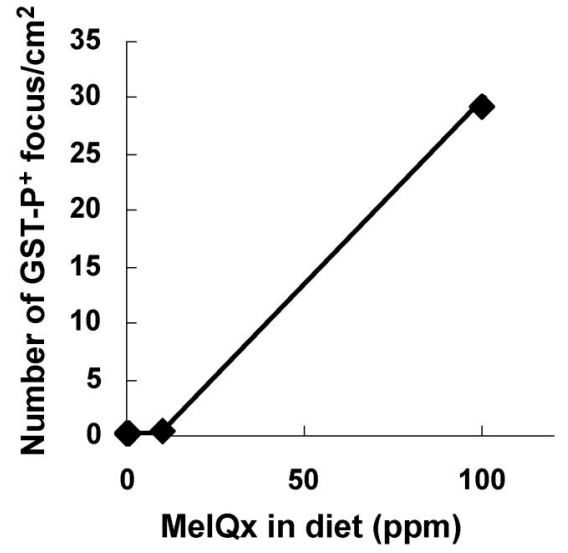

Fig. 3. Dose effect of MeIQx on liver preneoplastic changes. GST ${ }^{+}$ focus numbers per $\mathrm{cm}^{2}$ was measured after 16-week administration. Vertical bar indicates standard deviation. Data were from (6).

increase from the control group was observed. We reevaluated threshold possibilities using their data which did not show large coefficients of variation (less than 2), then plotted the findings on an arithmetic scale. The ACF number/rat used were $0.3 \pm 0.7,0.6 \pm 1.0,1.5$ \pm 1.4 , and $5.0 \pm 2.8$ at $0,50,100$, and $400 \mathrm{ppm}$, respectively. The data for these experimental groups were significantly different from that seen for the control animals, and a linear-no-threshold dose response, fitting to $\mathrm{y}=0.0123 \mathrm{x}+0.120, \mathrm{R}=0.998$ was observed (Fig. 2d). Supportive evidence for the presence of threshold for colon carcinogenesis was not obtained by the study of preneoplastic changes.

MelQx-liver tumorigenesis: Kushida et al. reported that liver tumors were induced in F344 male rats by feeding diets containing MeIQx for 52 weeks (17). Tumor (adenoma or carcinoma) incidences were $0 \%, 17$ $\%, 90 \%$ and $100 \%$ at $0,100,200$, and $400 \mathrm{ppm}$, respectively. Since the experimental period was short compared to the life span, these data are not appropriate for analysis of mode of dose response. Murai et al. fed MeI$\mathrm{Qx}$ for 104 weeks $(\mathrm{n}=51)(5)$. Tumor incidences were 0 , 0,0 , and $39 \%$, with $0,0.001,1$ and $100 \mathrm{ppm}$, respectively. Because the dosing spaces were so large, no information about mode of dose response was obtained.

MelQx-liver preneoplastic changes: As for preneoplastic changes, the dose response for glutathione $S$-transferase placental form (GST-P)-positive foci was analyzed using 150 rats (dosed with 0 or $1 \mathrm{ppm}$ ) or 50 rats (dosed with 10 or $100 \mathrm{ppm}$ ) for each group (6). We plotted the results on an arithmetic scale and confirmed that the dose response was non-linear (Fig. 3). The response rate changed greatly at around $10 \mathrm{ppm}$ and a threshold possibly exists. The study of preneoplastic changes indicates the possibility of presence of a threshold for liver tumorigenesis.
Table 1. Summary of treshold

\begin{tabular}{|c|c|c|c|}
\hline Chemical & Species & Organ & Threshold \\
\hline AAF & Mouse & Bladder & + \\
\hline AAF & Mouse & Liver & - \\
\hline PhIP & Rat & Mammary gland & - \\
\hline PhIP & Rat & $\begin{array}{l}\text { Systemic } \\
\text { (lymphocytic leukemia) }\end{array}$ & - \\
\hline $\mathrm{PhIP}$ & Rat & Colon & $+?$ \\
\hline MeIQx & Rat & Liver* & $+?$ \\
\hline
\end{tabular}

Presence or absence of threshold was estimated from line shape of dose response curve.

+ : Apparent threshold.

- : No threshold.

+ ?: Possible threshold.

*: Preneoplastic change

\section{Dose Response Mode Changes by Target Organs}

Table 1 shows a summary of the thresholds for AAF, PhIP, and MeIQx carcinogenesis. In the ED01 mouse study of AAF, non-linear dose response with an apparent threshold for bladder carcinogenesis was demonstrated, and linear-no-threshold dose response for liver carcinogenesis was noted under the same experimental conditions. In a PhIP study, liner-no-threshold dose response was suggested for mammary carcinogenesis and lymphocytic leukemogenesis. However, a nonlinear dose response was observed for colon carcinogenesis under the same experimental conditions. In a MeIQx carcinogenesis study, a non-linear dose response was observed for liver preneoplastic changes. The possibility of presence of a threshold remained when a non-linear dose response was observed (Table 1). It is suggested that a particular carcinogen's dose response changes by the target organs.

\section{Dose Response in Genotoxicity}

LacZ transgene mutations in the large intestine of mice after administration of 0,2 , or $20 \mathrm{mg} / \mathrm{kg} / \mathrm{day}$ of PhIP by gavage for 4 days (sacrificed 7 days after the last treatment) showed a linear-no-threshold dose response (18). Hoshi et al. (8) reported that MeIQx (after feeding to rats for 16 weeks) induced LacI mutation in the liver with nonlinear dose response and the presence of a threshold was shown by plotting on loglog scale without subtracting the background value. The doses tested were 0.001 to $100 \mathrm{ppm}$ in diet over 10 -fold spaces, with 10 control rats and 5 rats per experimental group. The average mutant frequencies increased dosedependently, but had large standard deviations. By plotting on an arithmetic scale, the shape of the dose response curves, either with full scales of $100 \mathrm{ppm}$ or 1 ppm (inset) did not show a threshold (Fig. 4a).

The dose response of two genotoxic agents, mitomycin $\mathrm{C}$ (MMC) and 1- $\beta$-D-arabinofuranosylcytosine (Ara-C), were reported following analysis of $10^{6}$ 

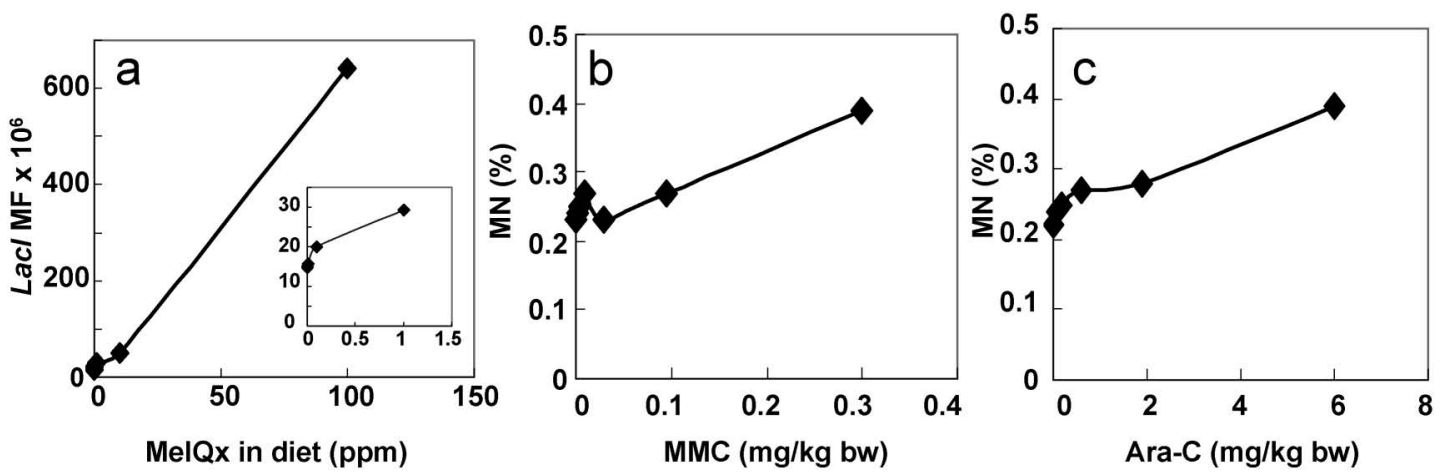

Fig. 4. Dose response of MeIQx, MMC, and Ara-C in genotoxicity in vivo. a: Big blue rats were fed diet containing various amounts of MeIQx for 16 weeks. LacI mutant frequency (MF) in the liver was determined. Data were from (8). b: Micronucleus (MN) induction in the peripheral blood reticulocytes of CD-1 mice by MMC administered by a single intraperitoneal injection. Data were from (9). c: MN induction in peripheral blood reticulocytes of CD-1 mice by various amounts of Ara-C administered by a single intraperitoneal injection. Data were from (9).

peripheral blood reticulocytes for micronucleus formation using flow cytometry (9). Although the presence of a practical threshold was reported by plotting on a loglog scale without subtracting background values (or on a semi-log scale), no threshold was indicated on an arithmetic scale (Figs. 4b and 4c). Thus, it may be considered that the genotoxic compound dose-response in vivo is generally linear-no-threshold.

However, two DNA-reacting genotoxic agents, methyl methanesulfonate and ethyl methanesulfonate were reported to show pragmatic thresholds for HPRT mutagenesis and micronucleus induction in human cells in vitro, although methylnitrosourea and ethylnitrosourea showed a linear-no-threshold dose response (10). The presence of a threshold for $N$-methyl- $N^{\prime}$ nitro- $N$-nitrosoguanidine (MNNG) mutagenesis has also been reported (11). In the case of MNNG, the threshold was demonstrated to be due to DNA repair action, using repair-proficient and repair-deficient strains of bacteria (11). Repair efficiency and the capacity of repair enzymes in this assay system were also estimated from the shapes of the dose response curves, and indicated that the repair was about $99.99 \%$ below the threshold (19).

Although MNNG genotoxicity dose response in vivo has not been demonstrated, it is thought that a threshold might be present for mutagenicity in vivo. It is worthwhile to confirm whether a threshold is present in MNNG carcinogenesis.

\section{Conclusion}

It has been demonstrated that the mode of carcinogen dose response changes depending upon the species, exposure chemical and target organ. The same chemical may show a linear-no-threshold dose response for one target organ even as it showed nonlinear dose response with an apparent or possible threshold in a different or- gan. It can be thought that for organs where background levels of tumor incidence are fairly high, carcinogens show linear dose responses, as observed in the mouse liver with AAF or in the rat mammary gland and hematopoietic system with PhIP. However, aflatoxin B1 showed a linear dose response in rat liver carcinogenesis (20), although the background level is extremely low. Considering the heterogeneity of the human species, it is wondered whether appropriate animal model systems are present for evaluation of the presence or absence of thresholds for carcinogenesis in humans. Further, the genotoxicity of DNA-reacting carcinogens generally shows a linear dose response in vivo. Therefore, it is reasonable to regulate genotoxic carcinogens based on the characteristics of linear-no-threshold. The U.S. Environmental Protection Agency recommends linear extrapolation as a default in carcinogen risk assessment (21). Application of the concepts of 'virtually safe dose' (VSD) or 'threshold of toxicological concern' (TTC) (22) would be feasible for regulation of very low doses of genotoxic carcinogens.

However, some types of genotoxic carcinogens show thresholds in genotoxicity in vitro, due to DNA repair. It could be that such types of carcinogens will show thresholds for carcinogenesis if the DNA repair enzymes similarly function in human cells. Such types of genotoxic carcinogens could be used safely below their threshold levels.

Acknowledgements: We thank Dr. Takashi Sugimura, President Emeritus, National Cancer Center and Dr. Takao Sekiya of Mitsubishi Kagaku Institute of Life Sciences, Tokyo, Japan for their critical reading of this manuscript. Dr. Mitsuko Masutani, Chief of Biochemistry Division, National Cancer Center Research Institute Tokyo, Japan is highly appreciated for her support of this study. The manuscript has been carefully proofread by an experienced medical editor whose first 
language is English. This study was supported by Grantin-Aid for the Health and Labour Sciences from the Ministry of Health, Labour and Welfare, Japan.

\section{References}

1 Gaylor DW. The ED01 study: Summary and conclusions. J Environ Pathol Toxicol. 1980; 3: 179-83.

2 Cairns T. The ED01 study: introduction, objectives, and experimental design. J Environ Pathol Toxicol. 1980; 3: $1-7$.

3 Poirier MC, Fullerton NF, Kinouchi T, Smith BA, Beland FA. Comparison between DNA adduct formation and tumorigenesis in livers and bladders of mice chronically fed 2-acetylaminofluorene. Carcinogenesis. 1991; 12: 895-900.

4 Fukushima S, Wanibuchi H, Morimura K, Iwai S, Nakae D, Kishida H, Tsuda H, Uehara N, Imaida K, Shirai T, Tatematsu M, Tsukamoto T, Hirose M, Furukawa F. Existence of a threshold for induction of aberrant crypt foci in the rat colon with low doses of 2-amino-1-methyl-6phenolimidazo[4,5-b]pyridine. Toxicol Sci. 2004; 80: 109-14.

5 Murai T, Mori S, Kang JS, Morimura K, Wanibuchi H, Totsuka Y, Fukushima S. Evidence of a thresholdeffect for 2-amino-3,8-dimethylimidazo-[4,5- $f$ ]quinoxaline liver carcinogenicity in F344/DuCrj rats. Toxicol Pathol. 2008; 36: 472-7.

6 Fukushima S, Wanibuchi H, Morimura K, Wei M, Nakae D, Konishi Y, Tsuda H, Uehara N, Imaida K, Shirai T, Tatematsu M, Tsukamoto T, Hirose M, Furukawa F, Wakabayashi K, Totsuka Y. Lack of a dose-response relationship for carcinogenicity in the rat liver with low doses of 2-amino-3,8-dimethylimidazo[4,5- $f$ ]quinoxaline or N-nitrosodiethylamine. Jpn J Cancer Res. 2002; 93: 1076-82.

7 Hasegawa R, Sano M, Tamano S, Imaida K, Shirai T, Nagao M, Sugimura T, Ito N. Dose-dependence of 2amino-1-methyl-6-phenylimidazo[4,5-b]-pyridine (PhIP) carcinogenicity in rats. Carcinogenesis. 1993; 14: 2553-7.

8 Hoshi M, Morimura K, Wanibuchi H, Wei M, Okochi E, Ushijima T, Takaoka K, Fukushima S. No-observed effect levels for carcinogenicity and for in vivo mutagenicity of a genotoxic carcinogen. Toxicol Sci. 2004; 81: 273-9.

9 Asano N, Torous DK, Tometsko CR, Dertinger SD, Morita T, Hayashi M. Practical threshold for micronucleated reticulocyte induction observed for low doses of mitomycin $\mathrm{C}$, Ara-C and colchicine. Mutagene- sis. $2006 ; 21: 15-20$.

10 Doak SH, Jenkins GJ, Johnson GE, Quick E, Parry EM, Parry JM. Mechanistic influences for mutation induction curves after exposure to DNA-reactive carcinogens. Cancer Res. 2007; 67: 3904-11.

11 Sofuni T, Nohmi T, Ohta T Hayashi M. Genotoxicity: Is a threshold concept applicable to evaluate the mutagenic activity of DNA-targeting substances!? Environ Mutagen Res. 2005; 27: 61-73.

12 Farmer JH, Kodell RL, Greenman DL, Shaw GW. Dose and time response model for the incidence of bladder and liver neoplasms in mice fed 2-acetylaminofluorene continuously. J Environ Pathol Toxicol. 1980; 3: 55-68.

13 Waddell WJ. Thresholds of carcinogenicity in the ED01 study. Toxicol Sci. 2003; 72: 158-63.

14 Hayes AW. Principles and Methods of Toxicology, $4^{\text {th }}$ ed. Taylor \& Francis, Philadelphia; 2001.

15 Ito N, Hasegawa R, Sano M, Tamano S, Esumi H, Takayama S, Sugimura T. A new colon and mammary carcinogen in cooked food, 2-amino-1-methyl-6phenylimidazo[4,5-b]pyridine (PhIP). Carcinogenesis. 1991; 12: 1503-6.

16 Kato T, Ohgaki H, Hasegawa H, Sato S, Takayama S, Sugimura T. Carcinogenicity in rats of a mutagenic compound, 2-amino-3,8-dimethylimidazo[4,5- $f$ ]quinoxaline. Carcinogenesis. 1988; 9: 71-3.

17 Kushida H, Wakabayashi K, Sato H, Katami M, Kurosaka R, Nagao M. Dose-response study of MeIQx carcinogenicity in F344 male rats. Cancer Lett. 1994; 83: 31-5.

18 Lynch AM, Gooderham NJ, Boobis AR. Organ distinctive mutagenicity in MutaMouse after short-term exposure to PhIP. Mutagenesis. 1996; 11: 505-9.

19 Watanabe M. Threshold-like dose-response relationships in a modified linear-no-threshold model: Application of experimental data and risk evaluation Genes Environ. 2008; 30: 17-24.

20 Wogan GN, Paglialunga S, Newberne PM. Carcinogenic effects of low dietary levels of aflatoxin B1 in rats. Food Cosmet Toxicol. 1974; 12: 681-5.

21 U.S. Environmental Protection Agency. Guidelines for carcinogen risk assessment; 2005 Mar. Report EPA/630/P-03/001F.

22 ILSI Europe: Threshold of toxicological concern (TTC): A tool for assessing substances of unknown toxicity present at low levels in the diet. By S Barlow. ILSI Europe Concise Monograph series. Brussels, Belgium. ISBN 1-57881-188-0 (2005). 